${ }^{1}$ Unidad de Adolescencia y Transición, Servicio de Pediatría, Hospital Dr. Luis Calvo Mackenna. Santiago, Chile.

2Departamento de Pediatría y Cirugía Infantil, Campus Oriente, Facultad de Medicina Universidad de Chile. Santiago, Chile. ${ }^{3}$ Centro de Salud del Adolescente SERJOVEN. Santiago, Chile. ${ }^{4}$ Centro de Adolescentes y Jóvenes, Departamento de Pediatría. Clínica Las Condes. Santiago, Chile.

Apoyo financiero: Ninguno.

Recibido el 26 de julio de 2015 , aceptado el 11 de mayo de 2016.

Correspondencia: Francisco Funes franciscofunes@gmail.com

\section{Conductas de riesgo en adolescentes con patología crónica compleja bajo control en un policlínico de un hospital pediátrico de Santiago}

\author{
FRANCISCO FUNES DÍAZ1, VERÓNICA GAETE PINTO' \\ Risk behaviors among adolescents \\ with complex diseases
}

Background: Adolescents with complex diseases may have a higher frequency of risk behaviors than their healthy peers. Aim: To characterize risk behaviors in adolescents with complex chronic diseases. Patients and Methods: Risk behaviors were assessed by means of a self-administered questionnaire designed for this purpose, in adolescents aged 10 to 19 years, who attended a pediatrics specialties clinic due to cystic fibrosis, congenital craniofacial anomalies, liver transplantation, kidney transplantation and spinal dysraphism. Results: We assessed 98 patients with a mean age of 14.1 years (59\% women). The most common behaviors were those related to accidents and violence, physical inactivity and unhealthy eating. Tobacco, alcohol and drug use, and sexual risk behaviors were observed in patients older than 15 years. Adolescents with congenital craniofacial anomaly followed by those with cystic fibrosis, showed a greater tendency towards some risk behaviors. Less physical activity and early onset of tobacco use predominated in women. Males had more physical violence. Eighteen percent of patients had more than 3 risk behaviors; $52 \%$ had two or three and $30 \%$ had one risk behavior. Conclusions: Risk behaviors in these adolescents with complex diseases varied according to the type of disease, gender and age.

(Rev Med Chile 2016; 144: 734-742)

Key words: Adolescent; Adolescent Behavior; Chronic disease; Risk-taking.
E xisten distintas definiciones de patología crónica, pero en general se refieren a una enfermedad que es diagnosticada en base a estándares profesionales, que dura años o décadas y que precisa un tratamiento regular. En los adolescentes, su prevalencia puede variar según la definición, pero comúnmente se considera que es del orden de 10 a $30 \%$ de la población entre 16 y 20 años $^{1,2}$. No se dispone de datos chilenos publicados al respecto y sólo es posible tener un acercamiento al referirnos a discapacidad, que entre los 15 y 29 años de edad, tiene una prevalencia de $4,8 \%{ }^{3}$.

En la adolescencia es común la presencia de conductas de riesgo (CR), definiéndose como aquellas actividades que incrementan las posibilidades de deteriorar el estado de la salud por morbilidad o mortalidad ${ }^{4}$. Existen 6 categorías de CR prioritarias según el Centro de Control y Prevención de Enfermedades de Estados Unidos de Norteamérica (CDC): uso de tabaco; consumo de alcohol y otras drogas; comportamientos sexuales que contribuyan a embarazos no deseados y a infecciones de transmisión sexual; comportamientos alimentarios no saludables; sedentarismo; comportamientos que favorezcan accidentes $y$ violencia ${ }^{5,6}$.

Se ha encontrado en algunos estudios que los adolescentes con condiciones crónicas de salud 
pueden presentar CR con mayor frecuencia que sus contrapartes sanos, evidenciando incluso, 3 o 4 CR a la vez. También pueden tener más problemas conductuales ${ }^{2,7}$. Ellos podrían temer ser excluidos del grupo de pares y sentir afectada su calidad de vida, por lo tanto, la presión por pertenecer al grupo puede impulsarles a realizar acciones poco saludables ${ }^{8,9}$. También es posible que algunos jóvenes con enfermedades crónicas pudieran tener más dificultades para integrarse a los grupos pro-sociales $^{10,11}$. Otros, al ver disminuida su expectativa de vida, pudieran elegir vivir la vida "al máximo", lo que incluiría la participación en una gama de comportamientos de riesgo para la salud ${ }^{2,12}$. Estas CR pueden permanecer en el tiempo, aun después de haber superado la enfermedad ${ }^{13,14}$. Los adolescentes con enfermedades crónicas están en doble desventaja ante las CR, ya que además del riesgo que conlleva la conducta por sí misma, se le suma el posible deterioro que le puede causar a su patología, por ejemplo, el hábito tabáquico en un paciente con fibrosis quística ${ }^{15}$.

En Chile se están implementando políticas de salud preventivas para adolescentes ${ }^{16,17}$. Sin embargo, no se conoce realmente cuál es el perfil de riesgo de los adolescentes que se atienden en los hospitales por patologías crónicas. El inicio de estudios que caractericen a esta población puede ser de gran utilidad para implementar programas hospitalarios en un ámbito integral y con la utilización racional de recursos.

El objetivo de este estudio es caracterizar CR prioritarias en salud, según el CDC, en adolescentes de entre 10 y 19 años con patología crónica compleja que son atendidos en un policlínico de especialidades de un hospital pediátrico.

\section{Pacientes y Métodos}

Se realizó un estudio descriptivo exploratorio transversal en los adolescentes de 10 a 19 años de edad que acudieron a control al Policlínico de Especialidades del Hospital Luis Calvo Mackenna, desde noviembre del año 2012 a noviembre del 2014, por padecer: fibrosis quística, malformación cráneofacial congénita, trasplante hepático, trasplante renal o disrrafia espinal. Se eligieron estas patologías por ser consideradas por los autores como relevantes en cuanto a complejidad, a necesidad de tratamientos constantes y a la cantidad de recursos, tanto personales como monetarios, invertidas en éstas.

Se excluyeron aquellos con déficit cognitivo evidente o que cursaban con alguna complicación aguda que interfiera la respuesta al cuestionario.

Se realizó una encuesta autoaplicada, pero con asistencia inicial, mostrando el cuestionario, preguntando acerca de su comprensión y aclarando dudas. Al finalizar la encuesta se revisó si todas las preguntas fueron respondidas.

Se procuró hacer la encuesta cuando acudieran a sus controles médicos habituales, hasta alcanzar el tamaño muestral.

El estudio se llevó a cabo siguiendo las normas de la Declaración de Helsinki y fue aprobado por el Comité de Ética Científico del Hospital Luis Calvo Mackenna.

\section{Instrumento de recolección de datos}

Se diseñó y validó un cuestionario para recopilar información sobre CR que el CDC monitorea por considerarlas relevantes para el desarrollo óptimo de la salud ${ }^{5,6}$. No se utilizaron encuestas nacionales ya validadas, dado que no englobaban el conjunto de CR que se deseaba estudiar, pero sí fueron una base del instrumento ${ }^{18-20}$ (Figura 1).

\section{Análisis estadístico}

Se realizó un análisis estadístico descriptivo. Para las variables continuas se aplicaron medidas de posición central: promedio y mediana; medidas de dispersión: desviación estándar y rango según distribución de la muestra. Las variables de tipo categóricas se presentaron en número absolutos y su frecuencia en porcentaje. Para determinar la presencia de asociación entre variables categóricas se aplicó la Prueba Exacta de Fisher. En aquellos factores que resultaron tener una asociación estadísticamente significativa se calculó el odds ratio (OR) mediante un análisis de regresión logística. Se consideró significativo un error alfa $<5 \%$. Para el análisis estadístico se utilizó el programa Stata v 12.1. El tamaño muestral mínimo calculado para encontrar las CR de menor frecuencia fue de 98 pacientes.

\section{Resultados}

En 2 años se reclutaron 98 pacientes. Sólo 3 rechazaron realizar la encuesta. Las características generales de los pacientes se detallan en la Tabla 1. 


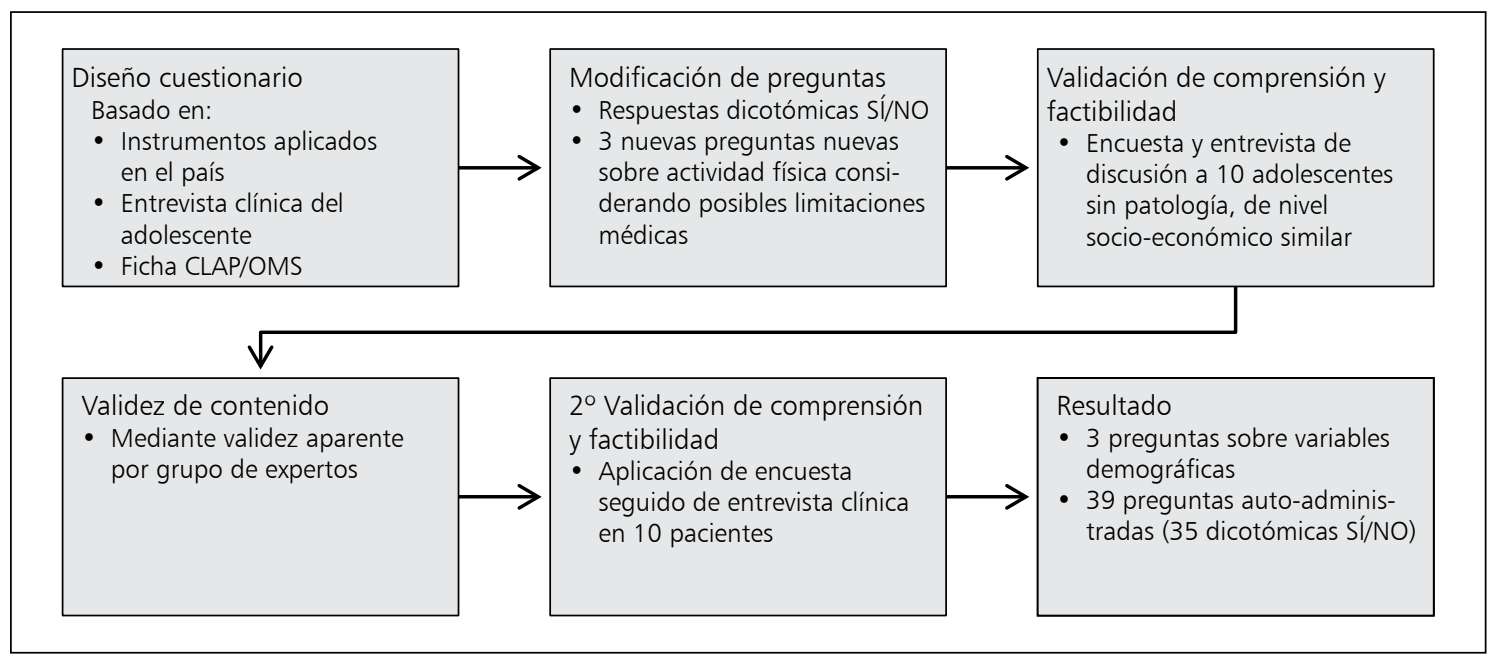

Figura 1. Diagrama de flujo de la validación del cuestionario.

El detalle de las CR y la relación por género se muestra en la Tabla 2. En la Figura 2, se muestra en orden decreciente la distribución de los 6 grupos de $\mathrm{CR}$, según número de pacientes que presentaron al menos 1 conducta del ítem.

Dentro del ítem accidentes y violencia, destaca que no hubo presencia de respuestas afirmativas relacionadas con asalto ni robo a casas. En los da-

Tabla 1. Características generales de los pacientes

\begin{tabular}{|lcc|}
\hline \multicolumn{1}{|c|}{$\mathbf{n}$} & $\%$ \\
Género & & \\
Masculino & 40 & 40,8 \\
Femenino & $58^{*}$ & 59,2 \\
Edad & & \\
10 a 14 años & 55 & 56,1 \\
15 a 19 años & 43 & 43,9 \\
Edad promedio (años) & & \\
Total & 14,1 & \\
Masculino & 14 & \\
Femenino & 14,2 & \\
Patología & & \\
Mielomeningocele & 26 & 26,5 \\
Trasplante renal & 26 & 26,5 \\
Trasplante hepático & 19 & 19,4 \\
Malformación cráneo-facial & 19 & 19,4 \\
Fibrosis quística & 8 & 8,2 \\
Total & 98 & 100 \\
\hline
\end{tabular}

*Mayor cantidad de mujeres entre los 10-14 años p 0,02. tos obtenidos en el ítem de sexualidad es necesario destacar que de los 10 adolescentes que tuvieron relaciones sexuales, sólo 2 utilizaron doble método de protección de embarazo (barrera y hormonal a la vez) y que los únicos que marcaron haber tenido sexo oral o anal son aquellos que habían tenido coito vaginal.

La menor actividad física y el inicio temprano de consumo de tabaco mostraron un predominio femenino. Los varones tuvieron más riñas.

\section{Conductas de riesgo y edad}

Los sujetos de 15-19 años tuvieron significativamente mayor frecuencia de CR relacionadas a alcohol y drogas, tabaco y actividad sexual que el grupo de 10-14 años (Tabla 3).

\section{Conductas de riesgo según patología}

Hubo diferencias significativas según enfermedad en los ítems accidentes y violencia y en sexualidad (Tabla 4). Respecto a cada conducta de riesgo en particular hubo diferencias significativas en la prevalencia de consumo en la vida de alcohol, pensamientos suicidas y manejo de vehículos motorizados sin tener licencia (Tabla 5).

\section{Relación entre conductas}

Todos marcaron haber tenido al menos 1 conducta de las preguntadas. La mayoría, 52\%, tuvo entre 2 y 3 conductas; $29,6 \%$ tuvo sólo 1 y $18,4 \%$ más de 3 . 
Tabla 2. Conductas de riesgo total y por género

\begin{tabular}{|c|c|c|c|c|c|}
\hline & $\begin{array}{c}\text { total } \\
(n=98)\end{array}$ & $\%$ & $\begin{array}{l}\text { Mujeres } \\
(n=58)\end{array}$ & $\begin{array}{l}\text { Hombres } \\
(n=40)\end{array}$ & $\mathbf{p}$ \\
\hline \multicolumn{6}{|l|}{ Tabaco } \\
\hline Prevalencia en la vida de tabaco & 17 & 17,4 & 11 & 6 & NS \\
\hline Edad de inicio promedio de tabaco (años) & 14 & - & 13 & 15 & $0,0299 *$ \\
\hline Prevalencia último mes de tabaco & 6 & $35,3^{\circ}$ & 5 & 1 & NS \\
\hline$\geq 1$ cigarrillo al día durante la última semana & 5 & $29,4^{\circ}$ & 4 & 1 & NS \\
\hline \multicolumn{6}{|l|}{ Alcohol y drogas } \\
\hline Prevalencia en la vida de alcohol & 26 & 26,5 & 14 & 12 & NS \\
\hline Edad de inicio promedio de ingesta alcohol (años) & 13,6 & - & 14,1 & 12,9 & NS \\
\hline Prevalencia último mes de alcohol & 7 & $25,9^{\circ}$ & 5 & 2 & NS \\
\hline Episodio de embriaguez & 8 & $28,6^{\circ}$ & 4 & 4 & NS \\
\hline$\geq 5$ vasos con alcohol en una salida & 5 & $17,9^{\circ}$ & 2 & 3 & NS \\
\hline Prevalencia de vida de marihuana & 7 & 7,1 & 4 & 3 & NS \\
\hline Edad al inicio promedio de fumar marihuana (años) & 14,8 & - & 15,3 & 14,3 & NS \\
\hline Prevalencia útimo mes de marihuana & 2 & $28,6^{\circ}$ & 2 & - & NS \\
\hline Prevalencia de vida otras drogas & 1 & 1 & 1 & - & NS \\
\hline \multicolumn{6}{|l|}{ Sexualidad } \\
\hline Relaciones sexuales & 10 & 10,2 & 8 & 2 & NS \\
\hline Relaciones sexuales sin condón & 8 & $80^{\circ}$ & 7 & 1 & NS \\
\hline Relaciones sexuales sin anticonceptivo & 5 & $50^{\circ}$ & 5 & - & NS \\
\hline Relaciones sexuales voluntarias $<15$ años & 5 & $50^{\circ}$ & 4 & 1 & NS \\
\hline Actividad sexual oral o anal & 4 & $40^{\circ}$ & 3 & 1 & NS \\
\hline \multicolumn{6}{|l|}{ Conductas alimentarias riesgosas } \\
\hline Atracón de comida & 34 & 34,7 & 24 & 10 & NS \\
\hline Ayuno $>8$ horas para bajar de peso & 23 & 23,5 & 18 & 5 & NS \\
\hline Provocarse vómitos para bajar de peso & 14 & 14,3 & 9 & 5 & NS \\
\hline Uso de medicamentos con el fin de controlar peso & 1 & 1 & 0 & 1 & NS \\
\hline Dejar de usar medicamentos para controlar peso & 2 & 2 & 1 & 1 & NS \\
\hline \multicolumn{6}{|l|}{ Inactividad física } \\
\hline No realiza educación física & 27 & 27,6 & 21 & 6 & $0,017^{*}$ \\
\hline No realiza ningún deporte fuera del colegio & 56 & 57,1 & 26 & 30 & $0,004^{*}$ \\
\hline Prohibición médica de realizar actividad física & 13 & 13,3 & 10 & 3 & NS \\
\hline \multicolumn{6}{|l|}{ Accidentes y violencia } \\
\hline Pensamientos suicidas & 29 & 29,6 & 21 & 8 & NS \\
\hline Intento suicida & 10 & 10,2 & 8 & 2 & NS \\
\hline Autoagresiones & 19 & 19,4 & 15 & 4 & NS \\
\hline Realizar bullying & 17 & 17,4 & 7 & 10 & NS \\
\hline Víctima de bullying & 25 & 25,5 & 16 & 9 & NS \\
\hline No uso de cinturón de seguridad & 38 & 38,8 & 26 & 12 & NS \\
\hline Manejar vehículos motorizados sin licencia & 11 & 11,2 & 5 & 6 & NS \\
\hline Ha peleado a golpes & 27 & 27,6 & 8 & 19 & $<0,01 *$ \\
\hline Porte de arma con intención de usarla & 2 & 2 & - & 2 & NS \\
\hline Realizar asalto & - & - & - & - & N/E \\
\hline Uso de arma en una pelea & 1 & 1 & - & 1 & NS \\
\hline Ha asaltado casas & - & - & - & - & $N / E$ \\
\hline
\end{tabular}

N/E: No estimable. NS: No significativo. ${ }^{\circ}$ Porcentaje de los que dieron respuestas positivas. *Significativo. 


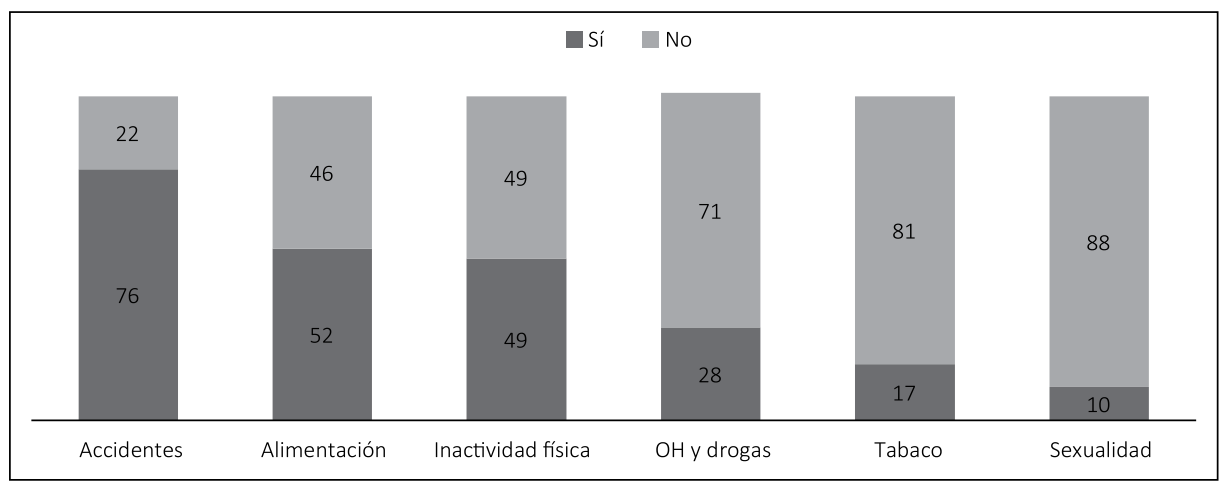

Figura 2. Presencia de al menos 1 conducta de riesgo en cada grupo.

Tabla 3. Conductas de riesgo según edad

\begin{tabular}{|lcccc|}
\hline Conducta de riesgo & $\mathbf{1 5}$ años & $>\mathbf{1 5}$ años & Total & p value \\
\hline Accidente y violencia & 41 & 35 & 76 & 0,472 \\
\hline Inactividad física & 30 & 19 & 49 & 0,416 \\
Conducta alimentaria & 31 & 21 & 52 & 0,542 \\
Alcohol y drogas & 11 & 17 & 28 & ${ }^{*} 0,029$ \\
Tabaco & 3 & 8 & 17 & ${ }^{*} 0,001$ \\
Sexualidad & 2 & & 10 & ${ }^{*} 0,02$ \\
\hline
\end{tabular}

*Significativo.

Tabla 4. Grupos de conductas con diferencias según patología

\begin{tabular}{|c|c|c|c|c|c|c|c|c|c|c|c|}
\hline \multirow[t]{2}{*}{$\begin{array}{l}\text { Conducta de } \\
\text { riesgo }\end{array}$} & \multicolumn{2}{|c|}{$\begin{array}{l}\text { Fibrosis } \\
\text { quística }\end{array}$} & \multicolumn{2}{|c|}{$\begin{array}{l}\text { Malformación } \\
\text { cráneofacial }\end{array}$} & \multicolumn{2}{|c|}{$\begin{array}{c}\text { Mielo } \\
\text { meningocele }\end{array}$} & \multicolumn{2}{|c|}{$\begin{array}{l}\text { Trasplante } \\
\text { hepático }\end{array}$} & \multicolumn{2}{|c|}{$\begin{array}{l}\text { Trasplante } \\
\text { renal }\end{array}$} & \multirow[t]{2}{*}{$\begin{array}{c}p \\
\text { value }\end{array}$} \\
\hline & n & $\%$ & $\mathbf{n}$ & $\%$ & $\mathbf{n}$ & $\%$ & $\mathbf{n}$ & $\%$ & $\mathbf{n}$ & $\%$ & \\
\hline Sexualidad & 1 & 12,5 & 3 & 15,8 & 1 & 3,9 & 2 & 10,5 & 3 & 12,5 & ${ }^{*} 0,005$ \\
\hline Accidente y violencia & 7 & 87,5 & 19 & 100 & 17 & 65,4 & 12 & 63,2 & 21 & 80,8 & ${ }^{*} 0,015$ \\
\hline $\begin{array}{l}\text { Pacientes encuestados } \\
\text { en cada patología }\end{array}$ & 8 & & 19 & & 26 & & 19 & & 26 & & \\
\hline
\end{tabular}

*Diferencias significativas entre los porcentajes de conductas según enfermedad.

Tabla 5. Conductas de riesgo específicas con diferencias según patología

\begin{tabular}{|c|c|c|c|c|c|c|c|c|c|c|c|}
\hline \multirow[t]{2}{*}{ Conducta de riesgo } & \multicolumn{2}{|c|}{$\begin{array}{l}\text { Fibrosis } \\
\text { quística }\end{array}$} & \multicolumn{2}{|c|}{$\begin{array}{l}\text { Malformación } \\
\text { cráneofacial }\end{array}$} & \multicolumn{2}{|c|}{$\begin{array}{c}\text { Mielo } \\
\text { meningocele }\end{array}$} & \multicolumn{2}{|c|}{$\begin{array}{l}\text { Trasplante } \\
\text { hepático }\end{array}$} & \multicolumn{2}{|c|}{$\begin{array}{l}\text { Trasplante } \\
\text { renal }\end{array}$} & \multirow[t]{2}{*}{$\begin{array}{c}p \\
\text { value }\end{array}$} \\
\hline & $\mathbf{n}$ & $\%$ & $\mathbf{n}$ & $\%$ & $\mathbf{n}$ & $\%$ & $\mathbf{n}$ & $\%$ & $\mathbf{n}$ & $\%$ & \\
\hline Prevalencia en la vida de alcohol & 4 & 50 & 11 & 57,9 & 4 & 15,4 & 2 & 10,5 & 5 & 19,2 & ${ }^{*} 0,003$ \\
\hline Pensamientos suicidas & 4 & 50 & 11 & 57,9 & 6 & 23 & 3 & 15,8 & 5 & 19,2 & $* 0,017$ \\
\hline $\begin{array}{l}\text { Manejar vehículos motorizados } \\
\text { sin licencia }\end{array}$ & 2 & 25 & 5 & 26,3 & 3 & 11,5 & 1 & 5,3 & - & - & ${ }^{*} 0,021$ \\
\hline $\begin{array}{l}\text { Pacientes encuestados en cada } \\
\text { patología }\end{array}$ & 8 & & 19 & & 26 & & 19 & & 26 & & \\
\hline
\end{tabular}

*Diferencias significativas entre los porcentajes de conductas según enfermedad. 


\section{Discusión}

El abordaje de la situación de los adolescentes con patología crónica se realiza generalmente bajo un enfoque no categorial, es decir, sin diferenciar entre tipos de enfermedades, lo que posibilita aprovechar la experiencia y potenciar los conocimientos sobre enfermedades poco frecuentes, evitando también la estigmatización de algunas enfermedades ${ }^{21}$. Sin embargo, nuestros resultados muestran que también sería de utilidad considerar el diagnóstico particular, ya que se encontraron diferencias entre las CR dependiendo de este. Esta es una información relevante y pudiese ser considerada como uno de los aportes de este trabajo. En este estudio encontramos que los pacientes portadores de malformaciones cráneo-faciales congénitas tuvieron consistentemente la mayor frecuencia de CR, tanto en el ítem de conductas favorecedoras de accidentes y violencias, como en el de las conductas sexuales riesgosas. También ocurrió esto en conductas específicas como: la prevalencia de vida de consumo de alcohol, pensamientos suicidas y manejar vehículos sin licencia. El segundo lugar en frecuencia de estas mismas conductas lo ocuparon los pacientes con fibrosis quística. Por otro lado, existieron patologías asociadas a menor frecuencia de CR. En el ítem de sexualidad, los encuestados con diagnóstico de mielomeningocele fueron los que tuvieron el último lugar. Lo mismo ocurrió en aquellos con trasplante hepático en relación al ítem de accidentes y violencias. La menor frecuencia en consumo de alcohol y pensamientos suicidas la tuvieron los pacientes con trasplante hepático. Los que menos manejaron sin licencia fueron aquellos con trasplante renal y hepático. Posiblemente, estas diferencias encontradas pudieran deberse a algunas limitaciones particulares de cada enfermedad, no obstante es necesario mayores estudios para explicar este fenómeno.

Todos los pacientes encuestados tuvieron en común la presencia de al menos $1 \mathrm{CR}$, esto puede relacionarse con la búsqueda de límites e identidad que ocurre a esta edad, más que al hecho de ser enfermos ${ }^{2}$. Por otro lado, la co-ocurrencia de más de $3 \mathrm{CR}$ en algunos adolescentes estudiados $(18,4 \%)$ pudiese indicar que, más allá de la crisis normativa, pudiese existir alguna alteración en su salud psico-emocional.

Con respecto a la prevalencia de CR según edad, como era de esperarse, se encontró que ser adolescente en su etapa inicial es un factor protector en varias de ellas, lamentablemente las CR relacionadas con alimentación no saludable, inactividad física, accidentes y violencia, no tuvieron esta diferencia según edad, encontrándose con la misma frecuencia en las etapas tempranas de la adolescencia

\section{Prevalencia de conductas de riesgo}

Aunque no es un objetivo de este estudio establecer diferencias entre los adolescentes con enfermedad crónica y la población general de adolescentes, es interesante hacer la comparación de algunos datos.

El consumo de tabaco, alcohol y otras drogas fue menor a lo encontrado en la población escolar de Chile. En ésta, el consumo en el último mes de alcohol y de tabaco es, respectivamente, 5 y 4 veces mayor a lo encontrado en nuestro grupo de estudio. El consumo de marihuana alguna vez en la vida también es mayor en la población escolar general $(37,2 \%$ vs $7,1 \%)$. En otras drogas, como cocaína y pasta base, sucedió lo mismo que lo anterior ${ }^{18}$.

La prevalencia de relaciones sexuales en adolescentes encuestados fue 5 veces menor a lo observado a nivel país ${ }^{22}$. Sin embargo, la mitad de ellos comenzó a tenerlas antes de los 15 años, siendo esto más precoz que lo hallado en la población general ${ }^{19}$. Aunque la cantidad de pacientes con relaciones sexuales en el grupo estudiado fue baja, la mayoría de los que las iniciaron no mostraron estar preparados, ya que no se protegieron adecuadamente.

Se encontró que la mitad de los encuestados incurrían en conductas alimentarias riesgosas. La información nacional nos indica que la anorexia nerviosa y la bulimia están presentes en $0,4 \%$ de la población entre 12 y 18 años y que $8,3 \%$ de las adolescentes está en riesgo de presentar estos trastornos ${ }^{23,24}$. Sin embargo, si bien las conductas estudiadas son base de trastornos de la alimentación, no son equivalentes. En cuanto a episodios de atracones de alimentos, en adolescentes norteamericanos sin patología crónica ha sido posible encontrar que $9,9 \%$ de las mujeres y 3,0\% de los varones los tenían, lo que es menor a lo encontrado en esta investigación ${ }^{25}$.

La prevalencia a nivel nacional de sedentarismo de tiempo libre en el grupo de 15-24 años es 
de $75,9 \%$. En el grupo estudiado esto fue menor, pero debe considerarse que la forma de preguntar fue distinta y que el rango de edad fue de 10 a 19 $\operatorname{años}^{20}$.

La mortalidad de los adolescentes por causas externas representa el $60 \%$ del total de las muertes en adolescentes ${ }^{26,27}$. Las ideas de terminar con la vida propia han sido encontradas en $30 \%$ de los adolescentes y la mitad de estos ha hecho algún intento para conseguirlo ${ }^{28}$. En nuestro estudio, la prevalencia de ideación suicida fue similar a la de la población adolescente general, aunque los intentos suicidas fueron menores. La prevalencia de jóvenes que se infligieron autoagresiones fue similar a la encontrada en población general de entre 14 y 17 años de la Región Metropolitana (19,2\%), pero mayor a lo encontrado en Inglaterra ${ }^{29-31}$. Ser víctima de bullying fue el doble a lo encontrado en un estudio en Chile, pero las peleas a golpes tuvieron similar frecuencia ${ }^{32}$.

En Suiza, Surís y cols. encontraron que los adolescentes con patología crónica tenían mayor cantidad de consumo de tabaco, alcohol, marihuana y otras drogas ilegales que el grupo control. En aquel estudio encontraron que 35\% realizaron actos antisociales, en cambio, en nuestros resultados sólo se encontró $1 \%{ }^{2}$. Esta discrepancia podría deberse a que en nuestra investigación sólo se estudiaron sujetos de menor edad y con patologías crónicas complejas específicas. En dicho estudio también se halló 7\% de conductas relacionadas con trastornos alimentarios, lo que es varias veces menor a $53 \%$ presente en nuestros resultados.

Tal vez, mientras mayor sea la complejidad de la enfermedad, exista una mayor supervisión de los padres, y por eso se encontró menor prevalencia de algunas conductas con un perfil disruptivo. Por otro lado, la prevalencia se mantuvo o incluso aumentó en conductas llamadas internalizantes (que son aquellas asociadas a retraimiento, ansiedad o depresión), como lo son las autoagresiones o los intentos extremos para bajar de peso. Esto se pudiera explicar dado que estas requieren un involucramiento en áreas que muchas veces no son tan evidentes para los padres o que pudiesen verse relegadas por temas más inmediatos, como los de la salud física ${ }^{33,34}$.

\section{Limitaciones}

Hubo un predominio de mujeres en adolescentes menores de 15 años, que no se explica por el azar, esto podría deberse un problema de la muestra, ya que no son patologías ligadas al sexo. Es llamativo que esta diferencia desaparezca en los mayores de 15 años.

Por otro lado, el haber utilizado un instrumento nuevo, limitó la comparación con la población general de adolescentes, sin embargo, no era parte de nuestros objetivos. Esta investigación no da información profunda sobre cada CR, no explica que hay detrás de estas, ni tampoco permite conocer si los adolescentes tenían comorbilidad psiquiátrica, pero sí permite tener la primera visión general sobre las $\mathrm{CR}$ en adolescentes con enfermedades crónicas atendidos en un centro de alta complejidad en nuestro país.

En conclusión, fue posible encontrar que todos los adolescentes con patología crónica tuvieron $\mathrm{CR}$, hallando con mayor frecuencia conductas predisponentes a accidentes y violencia y conductas alimentarias riesgosas. En el grupo estudiado la edad no fue un factor protector en las CR relacionadas con alimentación no saludable, inactividad física, accidentes y violencia, por otro lado, aunque existieron diferencias según género, estas fueron puntuales.

Finalmente, estimamos que es destacable encontrar que hubo diferencias en las CR que presentaban los adolescentes según tipo de patología que padecían.

Los hallazgos encontrados pueden ser de gran utilidad para plantear, con evidencia, la necesidad de realizar actividades preventivas. Estas podrían enfocarse en aquellas CR encontradas con mayor frecuencia a nivel general, o bien centradas en grupos particulares de pacientes en base a requerimientos específicos, pudiendo así optimizar recursos.

\section{Referencias}

1. Van der Lee JH, Mokkink LB, Grootenhuis MA, Heymans HS, Offringa M. Definitions and measurement of chronic health conditions in childhood: a systematic review. JAMA 2007; 297 (24): 2741-51.

2. Surís JC, Michaud PA, Akre C, Sawyer SM. Health risk behaviors in adolescents with chronic conditions. Pediatrics 2008; 122 (5): 1113-8.

3. FONADIS 2004. Primer estudio nacional de discapacidad en Chile. Disponible en: http://www.ine.cl/canales/ chile_estadistico/encuestas_discapacidad/pdf/presenta- 
cion.pdf [Consultado el 6 de Junio de 2015].

4. Keeler H J, Kaiser MM. An integrative model of adolescent health risk behavior. J Pediatr Nurs 2010; 25 (2): 126-37.

5. Eaton DK, Kann L, Kinchen S, Ross J, Hawkins J, Harris WA, et al. Youth risk behavior surveillance-United States, 2005. J Sch Health 2006; 76 (7): 353-72.

6. Newman K, Harrison L, Dashiff C, Davies S. Relaciones entre los tipos de padres y comportamientos de riesgo en la salud del adolescentes: una revisión bibliográfica integrada. Rev Latino-Am. Enfermagem 2008; 16: 14250.

7. Pinquart $\mathrm{M}$, Shen $\mathrm{Y}$. Behavior problems in children and adolescents with chronic physical illness: a meta-analysis. J Pediatr Psychol 2011; 36 (9): 1003-16.

8. Suris J, Michaud P, Viner R. The adolescent with a chronic condition. Part I: developmental issues. Arch Dis Child 2004; 89 (10): 938-42.

9. Avendaño M J, Barra E. Autoeficacia, apoyo social y calidad de vida en adolescentes con enfermedades crónicas. Terapia Psicológica 2008; 26(2): 165-72.

10. Patton GC, Carlin JB, Coffey C, Wolfe R, Hibbert M, Bowes G. Depression, anxiety, and smoking initiation: a prospective study over 3 years. Am J Public Health 1998;88 (10): 1518-22.

11. Valencia LS, Cromer BA. Sexual activity and other high-risk behaviors in adolescents with chronic illness: a review. J Pediatr Adolesc Gynecol 2000; 13 (2): 53-64.

12. Choquet M, Du Pasquier Fediaevsky L, Manfredi R. Sexual behavior among adolescents reporting chronic conditions: a French national survey. Adolesc Health 1997; 20 (1): 62-7.

13. Krull KR, Huang S, Gurney JG, Klosky JL, Leisenring W, Termuhlen A, et al. Adolescent behavior and adult health status in childhood cancer survivors. J Cancer Surviv 2010; 4 (3): 210-7.

14. Schultz KA, Chen L, Chen Z, Zeltzer LK, Nicholson HS, Neglia JP. Health and risk behaviors in survivors of childhood acute myeloid leukemia: a report from the Children's Oncology Group. Pediatr Blood Cancer 2010; 55 (1): 157-64.

15. Sawyer SM, Drew S, Yeo MS, Britto MT. Adolescents with a chronic condition: challenges living, challenges treating. Lancet 2007; 369 (9571): 1481-9.

16. MINSAL 2011. Sistematización de experiencias de salud con adolescentes. Disponible en: http://web.minsal.cl/ portal/url/item/bde734518e958ce3e040010164015a2a. pdf [consultado el 6 de junio de 2015].

17. MINSAL 2014. Orientacionestécnicas para el control de salud integral de adolescentes "Control Joven Sano". Disponible en: http://web.minsal.cl/sites/default/files/
files/2014CONTROLSALUDADOLESCENTE.pdf [Consultado el 6 de junio de 2015].

18. SENDA 2014. Décimo EstudioNacional de Drogas en Población Escolar de Chile. Disponible en: http://www. senda.gob.cl/wp-content/uploads/2014/07/decimo-escolares-2013.pdf [Consultado el 6 de junio de 2015].

19. INJUV 2011. Sexta Encuesta Nacional de Juventud. Disponible en: http://www.injuv.gob.cl/portal/wp-content/ files_mf/sextaencuestanacionaldejuventud.pdf [Consultado el 6 de junio de 2015].

20. MINSAL 2011. EncuestaNacional de Salud 2009-2010. Disponible en: http://web.minsal.cl/portal/url/item/ bcb03d7bc28b64dfe040010165012d23.pdf [Consultado el 6 de junio de 2015].

21. Stein RE, Jessop DJ. A non categorical approach to chronic childhood illness. Public Health Rep 1985; 97 (4): 354-62.

22. INJUV 2013. Séptima Encuesta Nacional de la Juventud. Disponible en: http://www.injuv.gob.cl/portal/wp-content/files_mf/septimaencuestanacionaljuventud2.pdf [Consultado el 8 de junio de 2015].

23. Vicente B, Saldivia S, De la Barra F, Melipillán R, Valdivia $\mathrm{M}, \mathrm{Kohn}, \mathrm{R}$. Salud mental infanto-juvenil en Chile y brechas de atención sanitarias. Rev Med Chile 2012; 140 (4): 447-57.

24. Correa M, Zubarew T, Silva P, Romero M. Prevalencia de riesgo de trastornos alimentarios en adolescentes mujeres escolares de la Región Metropolitana. Rev Chil Pediatr 2006; 77 (2): 153-60.

25. Neumark-Sztainer D, Wall M, Larson N, Eisenberg M, Loth K. Dieting and disordered eating behaviors from adolescence to young adulthood: findings from a 10year longitudinal study. J Am Diet Assoc 2011; 111 (6): 1004-11.

26. Romero M, Díaz F, Rubio P. Mortalidad por suicidio en adolescentes y jóvenes 1983-2003. Rev Chil Pediatr 2006; 77 (6): 623.

27. MINSAL 2010. Estrategia Nacional de Salud para el cumplimiento de los Objetivos Sanitarios de la Década 2011-2020. Disponible en: http://web.minsal.cl/portal/ url/item/c4034eddbc96ca6de0400101640159b8.pdf [Consultado el 6 de junio de 2015].

28. Ventura-Juncá R, Carvajal C, Undurraga S, Vicuña $P$, Egaña J, Garib MJ. Prevalencia de ideación e intento suicida en adolescentes de la Región Metropolitana de Santiago de Chile. Rev Med Chile 2010; 138 (3): 309-15.

29. Roizblatt A, Thomassen P, Pinedo M, Román P, Wolfenson A, Castillo-Carniglia A. Estudio de autoagresiones en un grupo de adolescentes de la Región Metropolitana en Chile. Rev GPU 2011; 7 (3): 324-29.

30. Stallard P, Spears M, Montgomery A, Phillips R, Sayal K. 
Self-harm in young adolescents (12-16 years): onset and short-term continuation in a community sample. BMC Psychiatry 2013; 13: 328 .

31. Kidger J, Heron J, Lewis G, Evans J, Gunnell D. Adolescent self-harm and suicidal thoughts in the ALSPAC cohort: a self-report survey in England. BMC Psychiatry 2012; 12: 69 .

32. WHO 2013. Global School-based Student Health Survey (GSHS) Chile, 2013 Fact Sheet. Disponible en: http://
www.who.int/chp/gshs/2013_Chile_GSHS_fact_sheet. pdf [Consultado el 6 de junio de 2015].

33. Ghandour RM, Kogan MD, Blumberg SJ, Perry DF. Prevalence and correlates of internalizing mental health symptoms among CSHCN. Pediatrics 2010; 125 (2): 269-77.

34. Barnes AJ, Eisenberg ME, Resnick MD. Suicide and self-injury among children and youth with chronic health conditions. Pediatrics 2010; 125 (5): 889-95. 